\title{
PAPEL DE LA BIBLIOTECA EN LA ENSEÑANZA DE COMPETENCIAS INFORMACIONALES EN POSGRADOS DE LA UNIVERSIDAD NACIONAL DE EDUCACIÓN A DISTANCIA (UNED)
}

Library roles in information literacy for postgraduate distance learning students at Universidad Nacional de Educación a Distancia (UNED)

Alexis Moreno-Pulido y Rosa Sánchez-Fernández

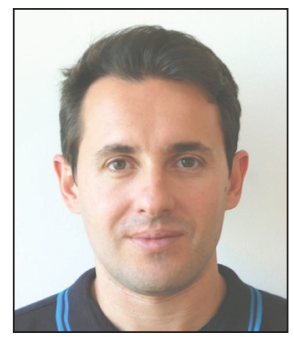

Alexis Moreno-Pulido es responsable de biblioteca en la Biblioteca del Campus Norte de la UNED donde desempeña funciones de formador en competencias informacionales en estudios adaptados al EEES y de apoyo a la investigación. Es licenciado en historia y en documentación por la Universidad de Salamanca. Ha trabajado en la Biblioteca del Instituto Cervantes de Manchester, en el Departamento de Estudios Europeos del Senado, en la Agencia Española del ISBN, en el Instituto Madrileño de Estudios Documentales y en la Biblioteca Nacional de España. Imparte regularmente talleres y conferencias y publica artículos sobre biblioteconomía y documentación.

http://orcid.org/0000-0003-1017-2976

axmoreno@pas.uned.es

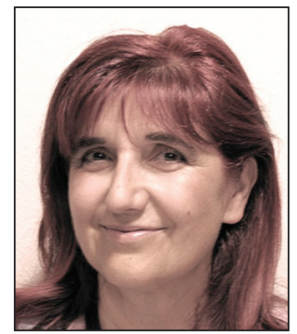

Rosa Sánchez-Fernández es coordinadora del Área de Apoyo a la Docencia y a la Investigación y de la Biblioteca del Campus Norte de la UNED donde realiza funciones de planificación, gestión y coordinación de equipos dedicados a la alfabetización informacional y al asesoramiento en investigación: estrategias de publicación, difusión, sexenios, etc. Es licenciada en historia y derecho por la UNED. Asesora al profesorado en materia de propiedad intelectual y uso legal de contenidos en cursos virtuales. Forma parte del consejo editorial de la revista Educación XX1 e imparte regularmente talleres y conferencias.

http://orcid.org/0000-0002-9286-6930

rmsanchez@pas.uned.es

Universidad Nacional de Educación a Distancia, Biblioteca del Campus Norte Juan del Rosal, 14. 28040 Madrid, España

\section{Resumen}

Se presenta la experiencia de la Biblioteca de la Universidad Nacional de Educación a Distancia (UNED) en la enseñanza a distancia de competencias informacionales en estudios oficiales de posgrado. Se detallan los niveles de actuación, estructura, contenidos, método, recursos de aprendizaje, medios de tutorización y se describen los ejercicios de evaluación. Se aborda la formación de equipos de trabajo y las funciones que realizan los bibliotecarios integrados en la formación. Finalmente se informa de los resultados de las encuestas de satisfacción y se discute la conveniencia de persistir en estas actividades formativas.

\section{Palabras clave}

Competencias informacionales; Alfabetización informacional; Bibliotecas universitarias; Bibliotecarios integrados; UNED, Enseñanza a distancia; Cursos virtuales; Doctorado.

\section{Abstract}

The library of the Spanish open university Universidad Nacional de Educación a Distancia (UNED) is involved in distance learning of information skills in formal postgraduate courses. This paper discusses action levels, structure, content, method, learning resources, tutoring, etc., and describes the student evaluation exercises. The formation of teams and the functions performed by embedded librarians is explained. Finally, the results of satisfaction surveys and the convenience to continue these training activities are discussed. 


\section{Keywords}

Information competencies; Information literacy; Academic libraries; Embedded librarians; UNED, Distance learning; Online courses; Doctorate.

Moreno-Pulido, Alexis; Sánchez-Fernández, Rosa (2015). “Papel de la biblioteca en la enseñanza de competencias informacionales en posgrados de la Universidad Nacional de Educación a Distancia (UNED)". El profesional de la información, v. 24, n. 5, pp. 665-673.

http://dx.doi.org/10.3145/epi.2015.sep.15

\section{Introducción}

Las bibliotecas universitarias han mostrado un interés creciente en la enseñanza de las competencias informacionales (en adelante $\mathrm{Cl}$ ). Esto se debe en parte a la implantación del Espacio Europeo de Educación Superior (en adelante EEES), que ha propiciado la participación de las bibliotecas universitarias en las actividades formativas de las universidades (Rebiun, 2007; 2012).

El EEES exige que los estudiantes sean formados en competencias transversales como gestión de la información o compromiso ético. Los universitarios encuentran dificultades para identificar sus necesidades de información, dedican demasiado tiempo a su búsqueda y recuperación, y obtienen generalmente resultados poco pertinentes (British Library; JISC, 2008). Tampoco manejan con solvencia los programas de gestión de la información ni disponen de criterios definidos para valorarla (Pinto-Molina; Puertas-Valdeiglesias, 2012; Pinto et al., 2013). Además desconocen las convenciones éticas y formales para elaborar trabajos académicos e ignoran el procedimiento de publicación científica (Gómez-Hernández, 2010). Se hace imprescindible por tanto que los alumnos adquieran $\mathrm{Cl}$ que reduzcan el tiempo dedicado al tratamiento y al uso de la información, para que puedan dedicarlo a labores discentes e investigadoras (Kingsley et al., 2011).

\section{El EEES exige que los estudiantes sean formados en competencias transver- sales como gestión de la información o compromiso ético}

La integración de las $\mathrm{Cl}$ en los planes de estudios persigue que el educando adquiera una comprensión sólida del proceso de búsqueda, evaluación y uso de la información científica, resaltando el marco legal y ético en que se produce y utiliza. Asimismo fomenta una actitud crítica que estimule la evaluación cualitativa de las fuentes de información, con el propósito de incorporar las más pertinentes y fiables al trabajo académico.

La incorporación de las $\mathrm{Cl}$ puede llevarse a cabo de diferentes formas (CRUE, 2012):

- con una asignatura específica y obligatoria;

- integrando los contenidos en varias asignaturas;

- mediante el reconocimiento de las competencias obtenidas por un organismo acreditado externo,

estableciendo cursos complementarios al plan de estudios.
En cualquiera de estos casos resulta necesaria la colaboración entre bibliotecarios y docentes universitarios en la planificación de los programas de enseñanza de $\mathrm{Cl}$ (Amante; Extremeño, 2012; Caridad-Sebastián; Martínez-Cardama, 2013), que deben ajustarse a los objetivos curriculares de los estudios oficiales en los que se integran.

Por su parte, la Biblioteca de la Universidad Nacional de Educación a Distancia (UNED) aspira a consolidarse "como colaboradora esencial en el proceso de enseñanza y aprendizaje, así como en la investigación y en la comunicación científica y convertirse en un líder innovador dentro de la comunidad universitaria" (Biblioteca UNED, 2012, p. 4). Con este propósito participa en la enseñanza a distancia de las $\mathrm{Cl}$ en estudios oficiales de grado, master y doctorado. La formación en $\mathrm{Cl}$ ayuda a que la biblioteca gane protagonismo como servicio universitario y estreche lazos con el profesorado.

\section{La integración de las $\mathrm{Cl}$ en los planes de estudios persigue que el educando ad- quiera una comprensión sólida del pro- ceso de búsqueda, evaluación y uso de la información científica}

\section{Objetivos}

El objetivo de este trabajo es presentar la implantación de los cursos de $\mathrm{Cl}$ en estudios oficiales de posgrado de la UNED, detallando niveles de integración, método, estructura, contenidos, recursos de aprendizaje, tutorización y el procedimiento de evaluación. Como objetivo específico se plantea el análisis de la satisfacción de los estudiantes con la formación recibida. Se discute asimismo la conveniencia de persistir en estas acciones formativas.

\section{Método}

El presente trabajo es un estudio descriptivo de una experiencia profesional en el que se analizan los cursos de $\mathrm{Cl}$ ofertados por la Biblioteca de la UNED.

Se examina en primer lugar la distribución de los cursos por niveles desde su inicio en el curso académico 2009/2010 hasta 2014/2015. En esta distribución se han comparado los datos de los posgrados y de los cursos de grado. Además se analiza la estructura de los cursos y se identifican los ejercicios prácticos sobre los que se fundamenta la evaluación de las $\mathrm{Cl}$. Asimismo se informa de la composición de los equipos de trabajo y se detallan las funciones de sus miembros. En una segunda etapa, se considera la satisfacción de los 
posgraduados con la formación recibida a partir de su respuesta en los cuestionarios aplicados tras la finalización de los cursos. Finalmente se reflexiona sobre la utilidad de persistir en estas acciones formativas.

\section{Descripción de la experiencia y resultados}

\subsection{Implantación}

La colaboración entre bibliotecarios y docentes en la formación de los estudiantes de posgrado existía con anterioridad a la implantación del EEES. La biblioteca participaba en las jornadas presenciales organizadas por los equipos docentes de los posgrados. Estas sesiones versaban sobre los recursos y servicios de la biblioteca para el apoyo al estudio y a la investigación. Sin embargo el alcance de estas actuaciones de formación de usuarios quedaba limitado a un conjunto de programas y llegaba a un reducido número de estudiantes.

La biblioteca aprovechó la estrecha relación con el personal docente para introducir la enseñanza de las $\mathrm{Cl}$ dentro de los nuevos planes de estudio. No obstante fue necesario vencer las resistencias planteadas tanto por algunos bibliotecarios, que se mostraron reacios a que la biblioteca asumiera nuevas tareas que pudieran desvirtuar las tradicionales funciones bibliotecarias, como por parte del profesorado, reticente acerca de la introducción de las $\mathrm{Cl}$ dentro del nuevo modelo de enseñanza.

La biblioteca apostó por presentar su contribución con un enfoque técnico y profesional, como una unidad experta en gestión de la información y dispuesta a colaborar con los equipos docentes. Además, desde la biblioteca se evitó equiparar la actividad de formación ejercida por los profesionales de la información con la llevada a cabo por el profesorado universitario. Esta estrategia permitió que la biblioteca fuera invitada a participar en las reuniones de las Comisiones Académicas que aprobaron los cursos de $\mathrm{Cl}$.

La experiencia piloto de formación en $\mathrm{Cl}$ en programas del EEES se llevó a cabo durante el curso académico 2009/2010 en tres masters de la Facultad de Psicología, y en tres más en la Facultad de Educación, Facultad de Filosofía y Escuela de Ingenieros respectivamente.

La biblioteca negoció con los responsables académicos de dichos posgrados la implementación de un curso virtual obligatorio denominado Competencias genéricas en información. En años posteriores se han ido incluyendo cursos virtuales en otras titulaciones de master y también en las enseñanzas oficiales de grado y doctorado. Además la biblioteca ha elaborado materiales a petición de los docentes para incorporarlos en sus asignaturas. En este caso las tareas de tutorización y de evaluación del aprendizaje recaen fundamentalmente en el docente, que remite dudas puntuales a los formadores de la biblioteca, mientras que en los cursos de la biblioteca son asumidas en su mayor parte por bibliotecarios. La tabla 1 muestra la evolución en el número de cursos de la Biblioteca de la UNED en estudios oficiales.

La Dirección de la Biblioteca creó en 2013 la Coordinación de Apoyo a la Docencia y a la Investigación con el objetivo de centralizar los esfuerzos de formación y de impulsar la participación de la biblioteca en un mayor número de pro- gramas. Desde 2013/2014 la biblioteca imparte un curso en la totalidad de los estudios de doctorado adaptados al EEES. El curso de $\mathrm{Cl}$ se incluye en las actividades formativas transversales que se basan en el artículo 4.1 del RD 99/2011 que regula las enseñanzas oficiales de doctorado. En 2014/2015 la biblioteca interviene en 20 masters: en 14 imparte un curso virtual y en 6 entrega documentos a los responsables de las asignaturas. Asimismo la biblioteca está presente en 11 grados: 2 cursos asistidos por bibliotecarios y en 9 se ceden materiales a los docentes.

Desde el curso académico 2013/2014 la biblioteca imparte un curso en la totalidad de los estudios de doctorado adaptados al EEES

\subsection{Método de enseñanza}

Los cursos están diseñados siguiendo la modalidad de enseñanza a distancia. El estudiante dispone online de toda la documentación que necesita para abordar el aprendizaje de modo autónomo. Este método de enseñanza consigue resultados óptimos de aprendizaje. Los niveles de adquisición de las $\mathrm{Cl}$ son ligeramente mejores que los obtenidos por la enseñanza presencial (Burkhardt; Kinnie; Cournoyer, 2008).

\subsection{Estructura}

La estructura de los cursos se ha adaptado a las enseñanzas.

\subsubsection{Master}

Se establecieron seis unidades didácticas agrupadas en dos niveles:

El primer nivel persigue facilitar la búsqueda y obtención de documentación pertinente, identificando las fuentes de información más adecuadas, y comprende tres unidades:

1. Determinar las necesidades de información.

2. Fuentes de información especializada.

3. Acceso a la información y al documento.

El segundo nivel está dedicado al tratamiento y ponderación de la información obtenida, y a su uso ético y legal. Consta también de tres unidades:

Tabla 1. Número de cursos de la biblioteca en estudios oficiales de la UNED.

\begin{tabular}{|l|c|c|c|}
\hline \multicolumn{1}{|c|}{ Curso académico } & Grado & Master & Doctorado \\
\hline $2009 / 2010$ & - & $6^{* *}$ & - \\
\hline $2010 / 2011$ & $2^{* *}$ & $6^{* *}$ & - \\
\hline $2011 / 2012$ & $2^{* *}$ & $6^{* *}$ & - \\
\hline $2012 / 2013$ & $2^{*} / 2^{* *}$ & $6^{* *}$ & \\
\hline $2013 / 2014$ & $7^{*} / 2^{* *}$ & $6^{*} / 13^{* *}$ & $17^{* *}$ \\
\hline $2014 / 2015$ & $9^{*} / 2^{* *}$ & $6^{*} / 14^{* *}$ & $18^{* *}$ \\
\hline
\end{tabular}

Nota: la oferta académica de estudios oficiales del EEES de la UNED en el curso académico 2014/2015 comprende 27 grados, 65 masters y 18 doctorados.

* Elaboración de materiales

** Curso virtual obligatorio de Competencias genéricas en información 
4. Gestión de la información.

5. Evaluación de la información.

6. Implicaciones éticas y legales del uso de la información.

\subsubsection{Doctorado}

Cuenta con diez unidades distribuidas también en dos niveles.

El nivel básico comprende seis temas:

1. Búsqueda bibliográfica.

2. Fuentes de información especializada: bases de datos y revistas electrónicas. Webs y repositorios institucionales.

3. Búsqueda y recuperación de la información. Interfaces de búsqueda y lenguajes de interrogación.

4. Herramientas de gestión de la información. Gestores bibliográficos. Estilos de publicación.

5. Evaluación de la información. Criterios de evaluación.

6. Uso ético y legal de la información.

Los doctorandos que han realizado un master que incluya un curso de $\mathrm{Cl}$ no necesitan realizar este nivel básico.

En el nivel avanzado, bloques 7 al 10, se aborda el proceso de publicación científica y se analizan las estrategias para compartir y promocionar la investigación. Además se incorpora un anexo que profundiza en el proceso de divulgación de la investigación científica.

7. El proceso de publicación científica (I).

8. El proceso de publicación científica (II).

9. Firmas personal e institucional de la producción científica.

10. Compartir y difundir la investigación.

Anexo: El proceso de publicación y difusión científica en profundidad.

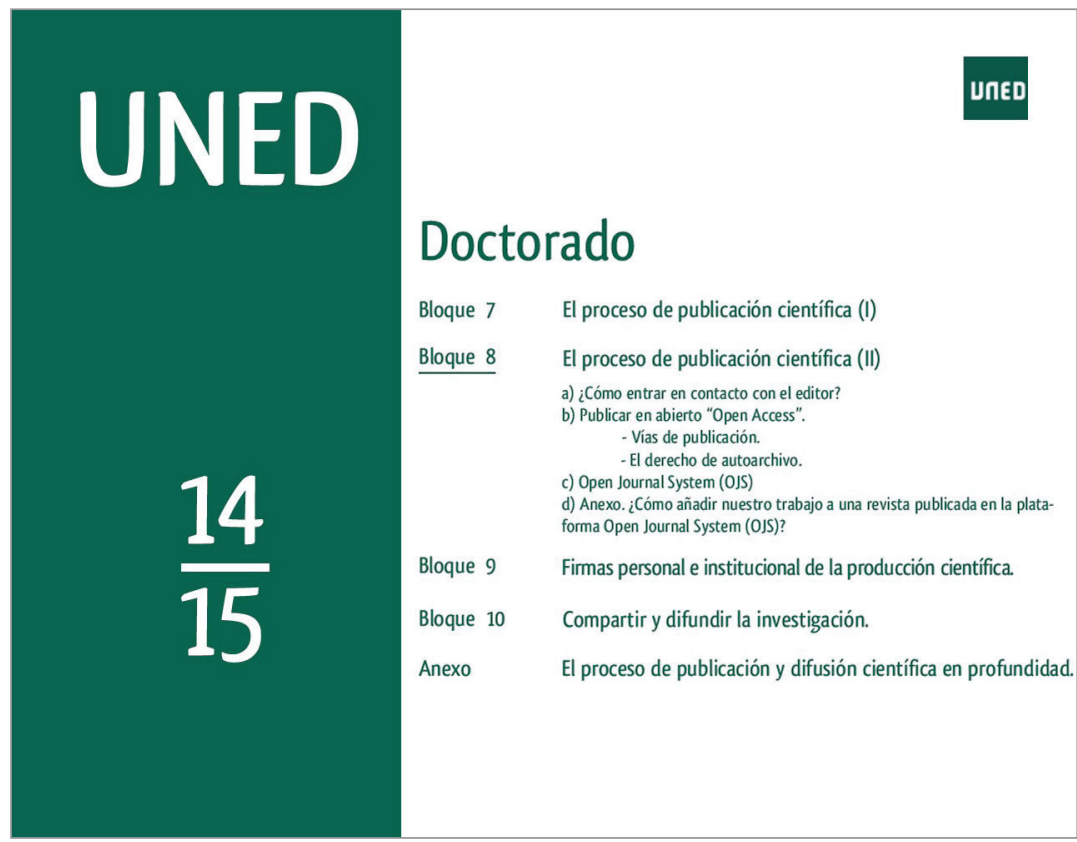

Figura 1. Portada del tema 8 del doctorado

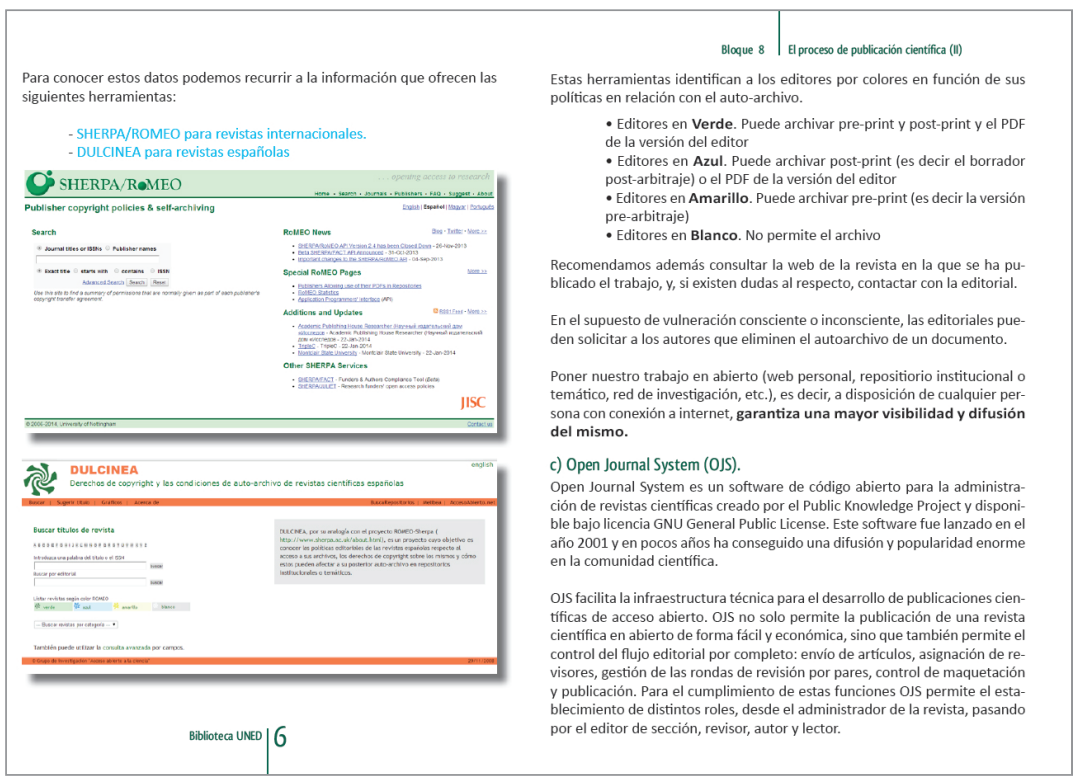

Figura 2. Página 6 del tema 8 del doctorado

\subsubsection{Grado}

El curso está formado por tres unidades didácticas y un anexo:

1. Fuentes de información.

2. Acceso a la información.

3. Gestión de la información.

Anexo. Uso ético y legal de la información.

El número de créditos ECTS (European credit transfer system) asignados a los cursos asciende a dos para los masters y uno para los grados. La formación transversal del doctorado no se estructura en créditos ECTS, pero tiene establecidas 50 horas de dedicación, en su nivel básico, y 30 horas más en el avanzado.

\subsection{Recursos de aprendizaje}

Los cursos virtuales están enriquecidos con los siguientes materiales:

- Unidades didácticas: en las que se especifican los objetivos de aprendizaje y se desarrollan los contenidos.

- Material complementario: en las unidades didácticas se incorporan enlaces a videoclases, talleres, tutoriales, guías de servicios, etc. El Centro de Medios Audiovisuales (Cemav) de la UNED produce las videoclases de la biblioteca, que se alojan tanto en el Canal UNED como en el Canal YouTube de ésta.

https://www.youtube.com/channel/UCV1Gvisq3Tnh_ a4dUNPOQIw 
El curso del doctorado se completa con la grabación de talleres prácticos sobre gestión, evaluación y difusión de la información, que se divulgan en el curso virtual.

- Actividades prácticas: en cada unidad didáctica se recomiendan prácticas para que el estudiante aplique los conocimientos adquiridos al análisis y reflexión de sus necesidades informativas y a la resolución de las mismas. Además se incorporan cuestionarios de autoevaluación (uno por unidad) para valorar su progreso. Por último, en los cursos virtuales se incluye una práctica obligatoria que será revisada por el equipo de formadores de la biblioteca.

\section{El Centro de Medios Audiovisuales (Cemav) de la UNED produce las vi- deoclases de la biblioteca}

\subsection{Tutorización}

Los participantes en los cursos virtuales disponen de un foro para enviar dudas y compartir información con sus compañeros, y pueden formular consultas individualizadas a través del correo electrónico y del teléfono. También se imparten tutorías presenciales en las bibliotecas de la sede central de la UNED en Madrid.

\subsection{Evaluación del aprendizaje}

La evaluación de las competencias adquiridas se realiza mediante actividades prácticas en las que los estudiantes deben resolver problemas de modo contextualizado y reflexivo. En ningún caso se examinan los conocimientos teóricos.

En los masters la evaluación se lleva a cabo mediante tres ejercicios:

En el primero, los alumnos deben crear una bibliografía conforme al manual de estilo de su especialidad utilizando un gestor bibliográfico. Este ejercicio requiere que el aspirante ejecute una búsqueda de información en una base de datos especializada sobre un tema de investigación planteado por el equipo docente. A continuación debe abrir un programa de gestión bibliográfica, capturar referencias de la fuente de información especializada y generar la bibliografía en un formato determinado. Por último se solicita la revisión de la bibliografía, la subsanación de posibles errores y la inclusión de pantallazos que ilustren el proceso seguido para resolver el problema.

En el segundo se aborda la inserción de citas y referencias en un manuscrito. Se presentan varios párrafos que simulan un trabajo académico y se indican los lugares en los que deben insertarse las citas. El grado de dificultad de esta práctica puede aumentar estableciendo distintos tipos de citas (autor en el texto, literal, etc.). Al final del manuscrito se deben agregar las referencias bibliográficas.

El gestor bibliográfico contratado es RefWorks y la aplicación para la inserción de citas y referencias Write-N-Cite-4. Lógicamente está permitido manejar otros gestores bibliográficos como Mendeley, Zotero, EndNote, etc. El propósito de este caso práctico es que los alumnos aprendan a citar las fuentes y evitar el plagio. En las unidades didácticas se aporta información sobre los estilos de publicación y la forma de generar citas y referencias, con o sin la mediación de un gestor bibliográfico.

El tercero consiste en ejecutar otra búsqueda de información sobre un tema propuesto por el equipo docente en una base de datos especializada, identificar cuáles son las revistas qué más artículos publican sobre el asunto, y argumentar sobre la conveniencia o no de publicar en dichas revistas, atendiendo a elementos como el factor de impacto, las tasas de aceptación/rechazo, el idioma de publicación, el número de artículos publicados sobre el tema en los últimos cinco años, etc. Esta práctica tiene como finalidad la identificación, mediante un proceso de evaluación cualitativa, de las mejores revistas para publicar una investigación.

En las correcciones se valora tanto el proceso como el resultado y las puntuaciones vienen acompañadas de comentarios individualizados. Los bibliotecarios elaboran informes para la evaluación de las competencias que son remitidos a los profesores.

En el doctorado, la evaluación de las competencias corresponde a las comisiones académicas de los programas de doctorado, previo informe remitido por la Coordinación de Apoyo a la Docencia y a la Investigación. Algunas de las actividades acometidas por los estudiantes son similares a las descritas antes, y otras profundizan en el networking y en la difusión del trabajo científico en redes de investigación como ResearchGate.

La evaluación de las competencias adquiridas se realiza mediante actividades prácticas en las que los estudiantes deben resolver problemas de modo contextualizado y reflexivo

\subsection{Equipos de trabajo}

La estructura de los equipos de trabajo ha sido diseñada por la Coordinación de Apoyo a la Docencia. Los grupos de trabajo están constituidos por miembros de la biblioteca, que asumen el papel de formadores y administradores del foro. En cada titulación están presentes dos formadores y un administrador del foro. Además existen tres figuras que centralizan una serie de tareas comunes para todas las titulaciones:
- administrador de cursos;
- administrador de datos;
- editor de textos.

Las funciones de los bibliotecarios que asumen estos roles son:

- Coordinador: negociar con el PDI (personal docente e investigador), planificar los cursos, distribuir cargas de trabajo y coordinar al grupo.

- Formador: redactar unidades didácticas, cuestionarios y prácticas.

- Administrador del foro: atender las dudas planteadas en el foro y elaborar el informe de actividad del mismo. 
- Administrador del curso: virtualizar los cursos, es decir, configurar la plataforma de enseñanza a distancia, alojar materiales, cuestionarios, prácticas, etc.

- Administrador de datos: realizar informes, tabular las encuestas de satisfacción, etc.

- Editor de textos: maquetar documentos.

El grupo de trabajo está integrado por 13 miembros (el 15\% del personal), clasificados en las siguien-

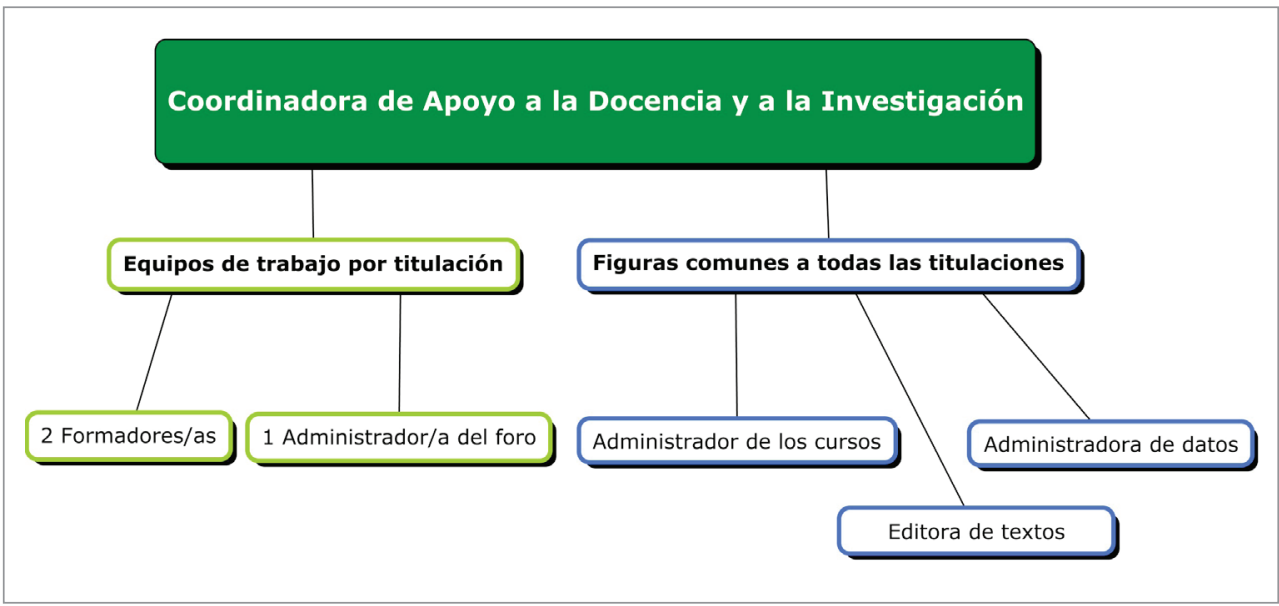

Figura 3. Organigrama de la Coordinación de Apoyo a la Docencia y a la Investigación.

tes categorías profesionales: un facultativo, un auxiliar con conocimientos de maquetación y 11 ayudantes, que dedican una media del $25 \%$ de su jornada laboral anual a trabajos de formación.

El trabajo se realiza de manera coordinada y colaborativa. El equipo ha utilizado espacios de trabajo comunes como la intranet de la biblioteca y servicios de almacenamiento en la nube como Dropbox.

\subsection{Evaluación y mejora continua de los cursos}

Los cursos virtuales son valorados mediante encuestas de satisfacción. El cuestionario consta de siete items de tipo

Likert de seis puntos: totalmente en desacuerdo, muy en desacuerdo, en desacuerdo, de acuerdo, muy de acuerdo, totalmente de acuerdo.

La formación en $\mathrm{Cl}$ ha cubierto las expectativas del $97,6 \%$ y del $95,1 \%$ de los estudiantes de master y doctorado respectivamente

En el curso académico 2013/2014 recibieron formación en $\mathrm{Cl} 444$ estudiantes de master y 245 doctorandos. En la figura 4 se presenta el grado de acuerdo y desacuerdo que manifestaron los alumnos de master $(\mathrm{N}=167)$ y doctorado $(\mathrm{N}=123)$, que cumplimentaron el cuestionario.

La formación en $\mathrm{Cl}$ ha cubierto las expectativas del $97,6 \%$ y del $95,1 \%$ de los estudiantes de master y doctorado respectivamente. Además el 98,2\% y el $93,5 \%$ de los mismos considera que el curso ha sido de utilidad para su formación profesional.

En la tabla 2 se desglosan por áreas temáticas las respuestas obtenidas en las dos preguntas examinadas con anterioridad.

La mitad de los encuestados de los masters de ciencias sociales y jurídicas y de humanidades y el $31,58 \%$ de sus homólogos de ciencias e ingenierías se muestran "totalmente de acuerdo" con la utilidad del curso para su formación profesional.

En el doctorado, el 58,9\% de los doctorandos de ciencias sociales y jurídicas, el 26,32 de los de ciencias e ingenierías y el 25,81 de los humanistas manifestaron su total conformidad con la

Figura 4. Grado de acuerdo y desacuerdo con los cursos de $\mathrm{Cl}$ 
utilidad de las enseñanzas.

En la encuesta se ha incluido un ítem adicional en escala del uno al seis para la valoración global del curso. La valoración media que los posgraduados han realizado de los cursos es de 5,12 sobre 6 . Especialmente representativa es la calificación media obtenida en los cursos de doctorado de ciencias sociales y jurídicas que se sitúa en 5,26. En la figura 5 se muestra la satisfacción global en cursos de posgrado por áreas temáticas.

Los resultados varían ligeramente entre las distintas áreas temáticas. Las mejores puntuaciones corresponden al área de ciencias sociales y jurídicas, mientras que las humanida-

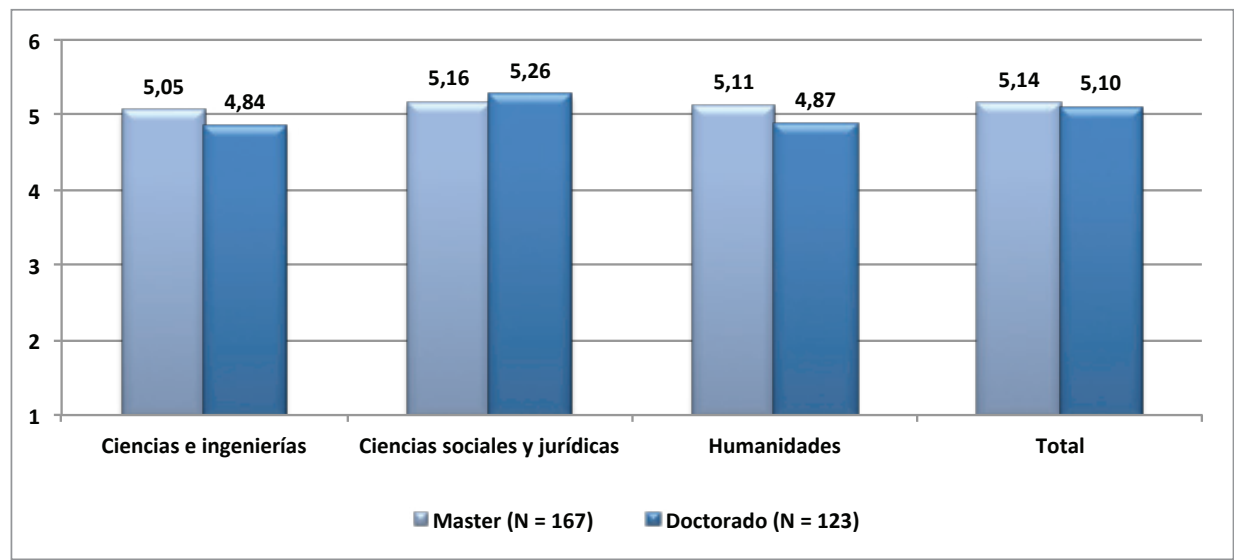

Figura 5. Satisfacción global con el curso por área de conocimiento

Tabla 2. Distribución de respuestas por áreas de conocimiento en master y doctorado.

\begin{tabular}{|c|c|c|c|c|c|c|c|}
\hline & & \multicolumn{3}{|c|}{ Master } & \multicolumn{3}{|c|}{ Doctorado } \\
\hline & & $\begin{array}{l}\text { Ciencias e } \\
\text { ingenierías }\end{array}$ & $\begin{array}{c}\text { Ciencias } \\
\text { sociales y } \\
\text { jurídicas }\end{array}$ & Humanidades & $\begin{array}{l}\text { Ciencias e } \\
\text { ingenierías }\end{array}$ & $\begin{array}{c}\text { Ciencias } \\
\text { sociales y } \\
\text { jurídicas }\end{array}$ & Humanidades \\
\hline & & $(\mathrm{N}=19)$ & $(\mathrm{N}=112)$ & $(\mathrm{N}=36)$ & $(\mathrm{N}=19)$ & $(\mathrm{N}=73)$ & $(\mathrm{N}=31)$ \\
\hline \multirow{12}{*}{$\begin{array}{l}\text { El curso ha cubierto } \\
\text { las expectativas }\end{array}$} & \multirow{2}{*}{$\begin{array}{l}\text { Totalmente en des- } \\
\text { acuerdo }\end{array}$} & \multirow{2}{*}{$0,00 \%$} & \multirow{2}{*}{$0,00 \%$} & \multirow{2}{*}{$0,00 \%$} & $5,26 \%$ & $4,10 \%$ & \multirow{2}{*}{$0,00 \%$} \\
\hline & & & & & $(\mathrm{N}=1)$ & $(\mathrm{N}=3)$ & \\
\hline & \multirow{2}{*}{ Muy en desacuerdo } & $5,26 \%$ & \multirow{2}{*}{$0,00 \%$} & \multirow{2}{*}{$0,00 \%$} & \multirow{2}{*}{$0,00 \%$} & $1,37 \%$ & \multirow{2}{*}{$0,00 \%$} \\
\hline & & $(\mathrm{N}=1)$ & & & & $(\mathrm{N}=1)$ & \\
\hline & \multirow{2}{*}{ En desacuerdo } & \multirow{2}{*}{$0,00 \%$} & $2,68 \%$ & \multirow{2}{*}{$0,00 \%$} & \multirow{2}{*}{$0,00 \%$} & $1,37 \%$ & \multirow{2}{*}{$0,00 \%$} \\
\hline & & & $(\mathrm{N}=3)$ & & & $(\mathrm{N}=1)$ & \\
\hline & \multirow{2}{*}{ De acuerdo } & $10,52 \%$ & $26,79 \%$ & $36,11 \%$ & $36,84 \%$ & $23,29 \%$ & $32,26 \%$ \\
\hline & & $(\mathrm{N}=2)$ & $(\mathrm{N}=30)$ & $(\mathrm{N}=13)$ & $(\mathrm{N}=7)$ & $(\mathrm{N}=17)$ & $(N=10)$ \\
\hline & \multirow{2}{*}{ Muy de acuerdo } & $52,64 \%$ & $32,14 \%$ & $36,11 \%$ & $26,32 \%$ & $26,03 \%$ & $35,48 \%$ \\
\hline & & $(\mathrm{N}=10)$ & $(\mathrm{N}=36)$ & $(\mathrm{N}=13)$ & $(\mathrm{N}=5)$ & $(\mathrm{N}=19)$ & $(\mathrm{N}=11)$ \\
\hline & \multirow{2}{*}{$\begin{array}{l}\text { Totalmente de } \\
\text { acuerdo }\end{array}$} & $31,58 \%$ & $38,39 \%$ & $27,78 \%$ & $31,58 \%$ & $43,84 \%$ & $32,26 \%$ \\
\hline & & $(\mathrm{N}=6)$ & $(\mathrm{N}=43)$ & $(\mathrm{N}=10)$ & $(\mathrm{N}=6)$ & $(\mathrm{N}=32)$ & $(\mathrm{N}=10)$ \\
\hline \multirow{11}{*}{$\begin{array}{l}\text { El curso ha sido de } \\
\text { utilidad para mi } \\
\text { formación laboral y } \\
\text { profesional }\end{array}$} & \multirow{2}{*}{$\begin{array}{l}\text { Totalmente en des- } \\
\text { acuerdo }\end{array}$} & \multirow{2}{*}{$0,00 \%$} & $0,89 \%$ & \multirow{2}{*}{$0,00 \%$} & $5,26 \%$ & $1,37 \%$ & \multirow{2}{*}{$0,00 \%$} \\
\hline & & & $(\mathrm{N}=1)$ & & $(\mathrm{N}=1)$ & $(\mathrm{N}=1)$ & \\
\hline & Muy en desacuerdo & $0,00 \%$ & $0,00 \%$ & $0,00 \%$ & $0,00 \%$ & $0,00 \%$ & $0,00 \%$ \\
\hline & \multirow{2}{*}{ En desacuerdo } & \multirow{2}{*}{$0,00 \%$} & $1,78 \%$ & \multirow{2}{*}{$0,00 \%$} & $10,52 \%$ & $2,74 \%$ & $6,45 \%$ \\
\hline & & & $(\mathrm{N}=2)$ & & $(\mathrm{N}=2)$ & $(\mathrm{N}=2)$ & $(\mathrm{N}=2)$ \\
\hline & \multirow{2}{*}{ De acuerdo } & $26,31 \%$ & $25,01 \%$ & $30,56 \%$ & $10,52 \%$ & $13,70 \%$ & $29,03 \%$ \\
\hline & & $(\mathrm{N}=5)$ & $(\mathrm{N}=28)$ & $(\mathrm{N}=11)$ & $(\mathrm{N}=2)$ & $(\mathrm{N}=10)$ & $(\mathrm{N}=9)$ \\
\hline & \multirow{2}{*}{ Muy de acuerdo } & $42,11 \%$ & $22,32 \%$ & $19,44 \%$ & $47,38 \%$ & $23,29 \%$ & $38,71 \%$ \\
\hline & & $(\mathrm{N}=8)$ & $(\mathrm{N}=25)$ & $(\mathrm{N}=7)$ & $(\mathrm{N}=5)$ & $(\mathrm{N}=17)$ & $(\mathrm{N}=12)$ \\
\hline & \multirow{2}{*}{$\begin{array}{l}\text { Totalmente de } \\
\text { acuerdo }\end{array}$} & $31,58 \%$ & $50,00 \%$ & $50,00 \%$ & $26,32 \%$ & $58,90 \%$ & $25,81 \%$ \\
\hline & & $(\mathrm{N}=6)$ & $(\mathrm{N}=56)$ & $(\mathrm{N}=18)$ & $(\mathrm{N}=9)$ & $(\mathrm{N}=43)$ & $(\mathrm{N}=8)$ \\
\hline
\end{tabular}

Nota: los porcentajes más elevados se muestran sombreados

des y las ciencias e ingenierías presentan valoraciones levemente inferiores.

A través de los foros y del correo electrónico se recogen las 
sugerencias, reclamaciones, agradecimientos y felicitaciones. En los informes del foro se presta especial atención a las incidencias ocurridas y a las sugerencias de mejora comunicadas tanto por los alumnos como por el administrador del mismo. Los informes sobre la actividad del foro y las encuestas de satisfacción sirven de base para la mejora continua de los cursos de la biblioteca.

\section{Discusión y conclusiones}

La principal conclusión es que el programa de formación a distancia en competencias informacionales cumple las expectativas de los estudiantes de posgrado y contribuye a mejorar la percepción de los servicios de formación ofertados por la Biblioteca de la UNED.

La valoración de los estudiantes es altamente positiva y refuerza la posición de la biblioteca como elemento de apoyo al aprendizaje. Los profesores también aportan un feedback alentador, que no obedece únicamente a la delegación de carga lectiva en terceros. Prueba de ello es la inclusión de material de $\mathrm{Cl}$ en asignaturas impartidas por docentes, con la participación puntual de bibliotecarios. Asimismo, las felicitaciones y agradecimientos recibidos sitúan el Área de Apoyo a la Docencia y a la Investigación por encima del resto de áreas y servicios.

Los informes sobre la actividad del foro y las encuestas de satisfacción sirven de base para la mejora continua de los cursos de la biblioteca

La integración de los bibliotecarios en tareas formativas contribuye a que se establezcan nuevos puentes de colaboración con el profesorado. Por ejemplo, la implantación de los cursos de $\mathrm{Cl}$ ha acrecentado la intervención de bibliotecarios en conferencias, jornadas y mesas redondas, entre otras actividades. Esta situación se ha extendido a otros servicios de la universidad como el Cemav, posibilitando que la biblioteca disponga de un Canal YouTube para las videoclases. Los bibliotecarios han protagonizado las grabaciones y los profesionales del Cemav las han producido con tecnología Polimedia de la UPV. En resumen, los bibliotecarios están desempeñando tareas creativas que incrementan la visibilidad de la biblioteca y de la profesión bibliotecaria. https://www.youtube.com/watch?v=1f_hN5ghCfk

La biblioteca ha adquirido un compromiso permanente con la mejora continua de la calidad de la enseñanza. Las opiniones de los bibliotecarios y de los estudiantes, recogidas en los informes del foro y en las encuestas de satisfacción, contribuyen a perfeccionar la formación de las $\mathrm{Cl}$. Los primeros consideran que el funcionamiento de los cursos podría optimizarse mediante la creación de una rúbrica que especifique con detalle los indicadores de evaluación de las $\mathrm{Cl}$. Asimismo, se preguntan si la adquisición de estas competencias está relacionada con la obtención de mejores calificaciones en los trabajos académicos.

El futuro de las actividades formativas de la biblioteca pasa por diseñar un curso para atender a los más de 125.000 ma- triculados en grados. Los profesores han detectado carencias en la formación de los educandos en la gestión de la información o en cómo evitar el plagio. Sin embargo, la biblioteca se enfrenta a amenazas externas como la amortización de plazas o la reducida tasa de reposición de efectivos, y a debilidades internas como el voluntarismo (no todos los bibliotecarios que participan en formación están adscritos a la Coordinación de Apoyo a la Docencia). Esta situación podría comprometer la extensión de las $\mathrm{Cl}$ a un mayor número de programas.

La supervivencia de las bibliotecas universitarias pasa por brindar servicios de valor añadido a las instituciones en las que se integran

La biblioteca también se ocupa de la formación en competencias digitales, participando en un curso online masivo y abierto (COMA) titulado Competencias digitales básicas, que está siendo revisado para su relanzamiento.

https://www.miriadax.net/web/comp_dig_basicas

La supervivencia de las bibliotecas universitarias pasa por brindar servicios de valor añadido a las instituciones en las que se integran. Anglada (2014) afirma que los bibliotecarios debemos establecer un nuevo paradigma de biblioteca basado en la función de apoyar en el manejo de la información y su transformación en conocimiento.

Los cursos de $\mathrm{Cl}$ entroncan con las funciones propias de la universidad frente a otras tareas bibliotecarias que pasan más desapercibidas. Además la implicación de las bibliotecas universitarias en la enseñanza de $\mathrm{Cl}$ brinda la oportunidad de publicitar otros productos y servicios bibliotecarios $y$, en consecuencia, mejorar las ratios de uso de los mismos.

\section{Agradecimientos}

Al grupo de trabajo de cursos virtuales en enseñanzas regladas: Ana-Luisa Sainz, Carolina Corral, Concepción Goizueta, Elodia Hernandez, Flora Sanz, Hugo Contreras, José-Antonio Vargas, Laura Martín, Leire Frisuelos, Paloma Sanz y Pilar González-Cremona.

\section{Bibliografía}

Amante, María-João; Extremeño Ana (2012). "Bibliotecarios universitarios - Profesores. ¿Caminos convergentes?". Revista española de documentación científica, v. 35, n. 2, pp. 298- 324.

http://dx.doi.org/10.3989/redc.2012.2.849

Anglada, Lluís (2014). "Are libraries sustainable in a world of free, networked, digital information?". El profesional de la información, v. 23, n. 6, pp. 603-611.

http://dx.doi.org/10.3145/epi.2014.nov.07

Biblioteca UNED (2012). Plan estratégico 2012/2015. http://portal.UNED.es/pls/portal/docs/PAGE/UNED_MAIN/ BIBLIOTECA/NORMATIVA/PLANESTRATEGICOBIBLIOTECA12_15. PDF 
British Library; JISC (2008). "Informe Ciber. Comportamiento informacional del investigador del futuro". Anales de documentación, v. 11, pp. 235-258.

http://revistas.um.es/analesdoc/article/viewFile/24921/24221

Burkhardt, Joanna M.; Kinnie, Jim; Cournoyer, Carina M. (2008). "Information literacy successes compared: online vs. face to face". Journal of library administration, v. 48, n. 3-4, pp. 379-389.

http://dx.doi.org/10.1080/01930820802289425

Caridad-Sebastián, Mercedes; Martínez-Cardama, Sara (2013). "El bibliotecario integrado en el aprendizaje universitario". El profesional de la información, v. 22, n. 2, pp. 149-154. http://dx.doi.org/10.3145/epi.2013.mar.09

CRUE (2012). Competencias informáticas e informacionales (CI2) en los estudios de grado.

http://www.uv.es/websbd/formacio/ci2_estudios_grado.pdf

España (2011). "Real decreto 99/2011, de 28 de enero, por el que se regulan las enseñanzas oficiales de doctorado". Boletín oficial del Estado, 10 de febrero, n. 35, pp. 13.90913.926.

http://www.boe.es/boe/dias/2011/02/10/pdfs/BOE-A-2011-2541. $p d f$

Gómez-Hernández, José-Antonio (2010). “University libraries and the development of lecturers' and students' information competencies". Revista de universidad y sociedad del conocimiento, v. 7, n. 2, pp. 39-49.

http://dx.doi.org/10.7238/rusc.v7i2.980

Kingsley, Karl; Galbraith, Gillian M.; Herring, Matthew; Stowers, Eva; Stewart, Tanis; Kingsley, Karla V. (2011). "Why not just Google it? An assessment of information literacy skills in a biomedical science curriculum". BMC medical education, v. 11, pp. 1-8.

http://dx.doi.org/10.1186/1472-6920-11-17

Pinto-Molina, María; Fernández-Ramos, Andrés; SánchezAmbriz, Gerardo; Meneses, Grizly (2013). "Information competence of doctoral students in information science in Spain and Latin America: A self-assessment". The journal of academic librarianship, v. 39, n. 2, pp. 144-154. http://dx.doi.org/10.1016/j.acalib.2012.08.006

Pinto-Molina, María; Puertas-Valdeiglesias, Susana (2012). "Autoevaluación de la competencia informacional en los estudios de Psicología desde la percepción del estudiante". Anales de documentación, v. 15, n. 2, pp. 1-15. http://dx.doi.org/10.6018/analesdoc.15.2.151661

Rebiun (2007). II Plan estratégico 2007-2010.

http://www.rebiun.org/documentos/Documents/IIPE/II\%20 Plan\%20Estrat\%C3\%A9gico.pdf

Rebiun (2012). III Plan estratégico de Rebiun 2020.

http://www.mcu.es/bibliotecas/docs/MC/ConsejoCb/CTC/ Bib_Univ/Planestrategico2020.pdf

\section{Inforảrea}

\section{Ayudamos a tu organización en la transformación digital y el gobierno de la información}

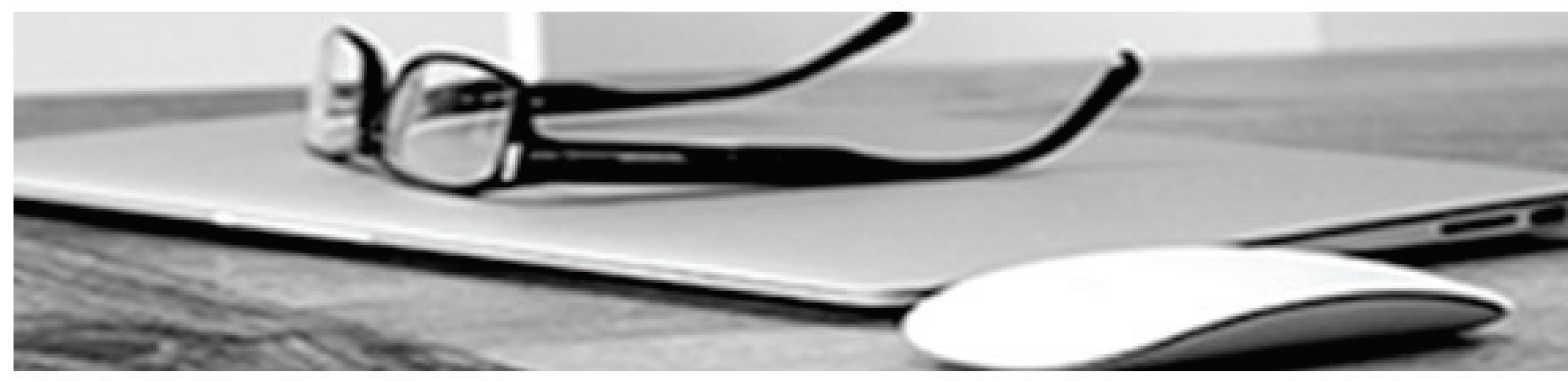

* Consultoría estratégica en gestión y gobierno de la información

* Gestión documental y "records management"

* Gestión de contenidos, intranets corporativas y entornos de colaboración

* Estudios especializados

Clientes satisfechos, cientos de empresas nacionales e internacionales y más de 30 años de experiencia son la mejor garantía de nuestra reputación. 